\title{
Regulation of Gratuitous $\beta$-Galactosidase Synthesis in Aerobacter aerogenes during an Adaptive Process
}

\author{
By N. RICHARDS \\ Department of Applied Sciences, Norwich City College, Norwich, NOR ${ }_{7} \mathrm{D}$
}

(Accepted for publication 29 October 1968)

\begin{abstract}
SUMMARY
When cells of Aerobacter aerogenes, growing exponentially in either glucose + mineral salts medium, or lactose + mineral salts (being fully adapted to growth in lactose), are inoculated for the first time into maltose + mineral salts medium containing $10^{-3} \mathrm{M}$-methyl- $\beta$-D-thiogalactoside, a very large amount of $\beta$-galactosidase is synthesized gratuitously during the lag and early stages of growth in the new medium. The abnormally high enzyme levels are maintained for some time. This is a form of 'preferential synthesis', which can be ascribed to a failure of catabolite repression during exposure to an unfamiliar compound as source of carbon and energy. The extent of preferential synthesis is increased by starvation of the organisms prior to inoculation in maltose medium.

Similar experiments involving organisms partially or completely adapted to growth in maltose show a smaller degree, or complete absence, of preferential synthesis and enhanced enzyme levels. The efficiency with which maltose exerts the 'catabolite repression' effect is thus a function of the degree of adaptation of the organisms to maltose as sole carbon source.

It is suggested that this breakdown of catabolite repression, followed by its recovery during adaptation, could well be a useful general feature of the adaptive process.
\end{abstract}

\section{INTRODUCTION}

Catabolite repression has been found to be an important element in the regulation of the rate of synthesis of several microbial enzymes, both induced and constitutive. Early work (see Neidhardt \& Magasanik, I956; Neidhardt, 1960; Magasanik, I96I) led to the suggestion that the repression was caused by the accumulation of catabolic intermediates, resulting from a rate of catabolism which is excessive relative to the rate of anabolism.

Later work has tended to confirm this hypothesis, and has established a considerable amount of evidence concerning the operation of the effect. In particular, the repressor was shown to act during the stage of enzyme induction (i.e. specific messenger RNA formation) rather than that of actual protein synthesis (Nakada \& Magasanik, 1964). This suggested that there might be a close link between the molecule effecting catabolite repression, and that associated with the product of the $i$ (regulator) gene in normal induced enzyme synthesis. This question has not been completely resolved, but Loomis \& Magasanik (1964) found that $i^{-}$mutants were subject to catabolite repression, and subsequently (Loomis \& Magasanik, 1967) discovered a new gene, $C R$, a mutation in which led to insensitivity to catabolite repression in the lac operon.

An additional factor is the distinction between 'acute transient catabolite repres- 
sion', which is overcome by the organism after varying periods of time, and a less severe permanent repression which persists (Moses \& Prevost, 1966). The transient effect, as far as it affects $\beta$-galactosidase synthesis, appears to require a functional operator gene in the lac operon, but it is suggested that the permanent effect is nonspecific, not operating via the regulatory system of the lac operon (Palmer \& Moses, 1967).

In general it is true to say that an accumulation of catabolic intermediates, either by an increase in the rate of their formation or a restriction in the rate of their incorporation into cell macromolecules, increases the effectiveness of repression, and vice versa (see above references, and Prevost \& Moses (1967) and Clark \& Marr (1964)).

\section{METHODS}

\section{Organism and growth conditions}

The strains of Aerobacter aerogenes used in these experiments all had maximal growth rate when glucose was sole carbon source (doubling time approximately $35 \mathrm{~min}$.), in a mineral salts medium. They were variously adapted to lactose and/or maltose as sole carbon source, or unadapted to either, the exact state of adaptation being given in the text. The growth medium was as follows: 3 parts of a solution containing $10 \mathrm{~g}$. $/ 1$. of the appropriate sugar (in the text ' $G$ ' represents glucose, ' $M$ ' maltose and ' $\mathrm{L}$ ' lactose), 7 parts of a buffered mineral salts medium $(\mathrm{pH}=7 \cdot \mathrm{I} 2)$ having the composition: $\mathrm{Na}_{2} \mathrm{HPO}_{4}$. I2 $\mathrm{H}_{2} \mathrm{O}, \mathrm{I0} \cdot 0 \mathrm{~g}$./1.; $\mathrm{KH}_{2} \mathrm{PO}_{4}, \mathrm{I} \cdot 85 \mathrm{~g}$./1.; $\left(\mathrm{NH}_{4}\right)_{2} \mathrm{SO}_{4}$, $\mathrm{I} \cdot 56 \mathrm{~g}$. $/ 1$.; $\mathrm{MgSO}_{4}, 62 \mathrm{mg} . / 1 . ; \mathrm{FeSO}_{4} \cdot 7 \mathrm{H}_{2} \mathrm{O}, 0.32 \mathrm{mg}$./1. When it was desired to induce $\beta$-galactosidase gratuitously in a culture growing in a maltose medium, a sterile solution of methyl- $\beta$-D-thiogalactoside (MTG) in mineral salts solution was added, replacing the same volume of plain mineral salts, to give a final concentration of $\mathrm{IO}^{-3} \mathrm{M}-\mathrm{MTG}$ (M+MTG medium). Cells were grown at $40^{\circ}$ with forced aeration. For further details of culture methods see Richards \& Hinshelwood (I96I).

\section{Growth rate and enzyme assay}

The bacterial concentration of cultures was measured either using a Hilger 'Spekker' light absorptiometer, or at $540 \mathrm{~m} \mu$ in a 'Unicam' SP 600 spectrophotometer, using a I cm. cell. The cell concentration symbol, $M$, is proportional to cell mass per unit volume, although expressed as 'number of standard cells per $10^{-6} \mathrm{ml}$.' (for a discussion of this see Richards \& Hinshelwood, I96I).

The $\beta$-galactosidase activity of organisms was measured using $o$-nitrophenyl- $\beta$-Dgalactopyranoside (ONPG) as chromogenic substrate, according to the method given in Richards \& Hinshelwood (I96I). The symbol $\alpha_{s}$ represents the $\beta$-galactosidase activity of washed, ultrasonically disrupted organisms (for details, see Richards \& Hinshelwood, 1962), per unit mass of organisms.

$\alpha_{c}$ represents the $\beta$-galactosidase activity per unit volume of culture, i.e. $\alpha_{c}=\alpha_{s} M_{c}$, where $M_{0}$ is the concentration of organisms in the culture from which the sample was taken.

The symbol $\Delta$ is used to indicate the change in a property ( $\alpha$ or $M$ ) from the time of inoculation of a culture (i.e. the slope of a plot of $\Delta \alpha_{c} v . \Delta M$ is a measure of the differential rate of synthesis of $\beta$-galactosidase). 


\section{RESULTS}

Transfer of non-induced organisms to $M+M T G$ medium

\section{Organisms fully adapted to maltose}

If a strain of Aerobacter aerogenes, fully adapted to maltose as sole source of carbon and energy, is transferred to $M+M T G$ medium, $\beta$-galactosidase synthesis is induced. The induction occurs without any preferential synthesis. In fact, with [MTG] $=10^{-3} \mathrm{M}$ the initial differential synthetic rate of $\beta$-galactosidase is lower than the equilibrium value. Figure I shows this: $\alpha_{s}$ increases roughly linearly with number of cell divisions $(N)$ after addition of inducer. If the organisms were synthesizing $\beta$-galactosidase at a steady differential rate of $d \alpha_{c} / d M=55, \alpha_{s}$ would increase with $N$ in the manner shown by the broken line.

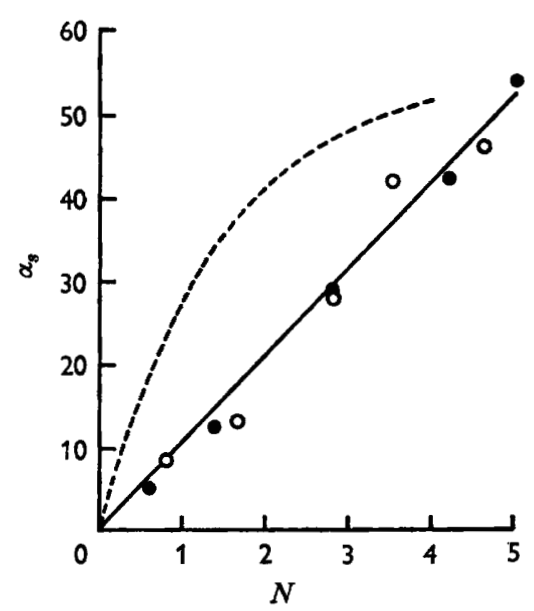

Fig. I. Induction of $\beta$-galactosidase by $10^{-3} \mathrm{M}-\mathrm{MTG}$ in Aerobacter aerogenes fully adapted to maltose, and growing in maltose medium. $N=$ number of generations after addition of inducer. $O, \Theta$, represent separate experiments. - - - : theoretical curve for a constant differential synthetic rate of $d \alpha_{c} / d M=55$.

A steady value of $d \alpha_{c} / d M$ has, however, been reached by $N=5$. When organisms were transferred from maltose medium to $\mathrm{M}+\mathrm{MTG}$, grown in this for 5 generations and then transferred to fresh $\mathrm{M}+\mathrm{MTG}$ medium, the plot of $\Delta \alpha_{c} v . \Delta M$ is subsequently a straight line of slope 5I. This is in good agreement with the value of $\alpha_{s}$ maintained during serial subculture in M+MTG medium. We can therefore accept $\alpha_{s} \approx 50$ as the equilibrium value during balanced growth.

\section{Organisms unadapted to maltose}

If an inoculum of organisms which have never been grown in maltose, nor induced with MTG, is taken from a glucose medium, washed, and added to a maltose medium, there is a lag of about $60 \mathrm{~min}$. After this, growth proceeds slowly until mass has increased about $50 \%$, when steady exponential growth follows, with mean generation time (m.g.t.) about 60 to $80 \mathrm{~min}$. MTG has no effect on growth. The mean generation time decreases steadily to a minimum over the course of several subcultures, but the bulk 
of the experiments reported below refer to the induction of $\beta$-galactosidase during this early period of lag and slow growth.

If the initial transfer from glucose, after washing, is to a $\mathbf{M}+\mathbf{M T G}$ medium, $\beta$-galactosidase induction follows the pattern shown in Fig. 2. Gratuitous induction of the enzyme and adaptation to growth in maltose occur simultaneously. It can be seen that $\beta$-galactosidase synthesis begins at zero time, and $\alpha_{s}$ rises linearly with respect to time, during the growth lag. This linear increase is continued beyond the point where growth begins, but is starting to level off at the end of the experiment. Accordingly, $\alpha_{c}$ rises linearly with respect to time before mass increase begins and more rapidly later. The plot of $\Delta \alpha_{c} v . \Delta M$ necessarily has apparently infinite slope at $t=0$ (Fig. 2c), since enzyme is synthesized without sensible mass increase. The slope of the plot falls gradually to a steady value of approximately 140 by $t=120$ minutes. This value is some 2.8 times the steady value of $d \alpha_{c} / d M$ reached during induction in
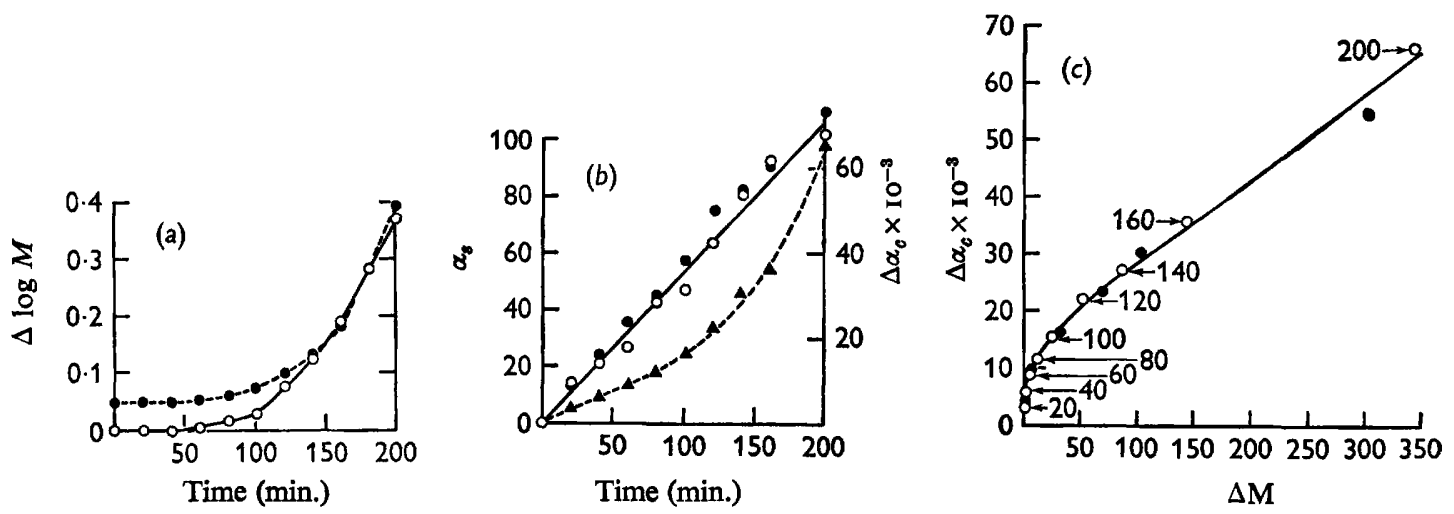

Fig. 2. Simultaneous induction of $\beta$-galactosidase and adaptation to maltose, of organisms never before exposed to either. (a) Growth, plotted as $\Delta \log M$, v. time: $\bigcirc-\bigcirc$ and - - represent separate experiments (with somewhat different initial cell concentrations). Growth approaches exponential towards the end of the experiment, with m.g.t. $\approx 65$ minutes. (b) Circles represent $\alpha_{g}$ for the corresponding culture in $(a)$. $\Delta---\Delta$ for culture indicated by closed circles. (c) Plot of $\Delta \alpha_{e} v . \Delta M$. Figures on curve are times after inoculation of culture $O$, in minutes.

maltose adapted cells. The maximum value of $\alpha_{s}$ is approximately twice the equilibrium value in adapted cells. This value is reached after $\mathrm{I} \cdot 3$ cell divisions in $\mathrm{M}+\mathrm{MTG}$ medium, and the 'equilibrium' value of $\alpha_{s}(\approx 50)$ is passed after 0.08 cell divisions.

This behaviour can be shown to represent a genuine preferential synthesis as follows. The alternative interpretation would be reincorporation of protein breakdown products into $\beta$-galactosidase molecules during protein turnover in the lag period, at the standard differential synthetic rate (see Mandelstam, 1957). This can be excluded because the turnover rate would have to be impossibly high to account for the large amount of enzyme synthesized. Suppose we plot the amount of enzyme synthesized per unit cell mass, $\Delta \alpha_{c} / M_{o}$, where $M_{s}=$ cell concentration at zero time, against time, during the lag and very early growth period (Fig. 3, curve A). The slope of this line, $(d / d t)\left(\Delta \alpha_{o} / M_{o}\right)$, subsequently referred to as $\epsilon$, is effectively the rate of enzyme synthesis per unit mass per unit time.

Suppose that the $\beta$-galactosidase is all produced as a result of protein turnover with 
reincorporation at the normal differential rate. If we define $\delta M$ as the amount of mass turnover per unit total mass in unit time, $\epsilon$ must be in the same ratio to $\delta M$ as is the small increment in $\alpha_{c}, d \alpha_{c}$, to the small increment in $M, d M$, when a steady value of the differential rate of synthesis has been reached in actively growing organisms,

i.e.

$$
\frac{\epsilon}{\delta M}=\left(\frac{d \alpha_{c}}{d M}\right) \text { equilibrium. }
$$

One remaining difficulty lies in choosing an appropriate equilibrium value for the differential rate of enzyme synthesis in the experiments under consideration, since for most of the experiment the cells are not growing exponentially. The terminal gradient of the $\Delta \alpha_{o} v . \Delta M$ plot in Fig. $2(c)$ gives the highest value which could be considered as an 'equilibrium' figure, since it is reached right at the beginning of exponential growth in maltose, about $3 \mathrm{hr}$ after the inoculation into maltose medium. For different experiments it lies within the range 120-140. From Fig. 3, curve A, $\epsilon=0.56$ enzyme units per unit mass per minute. Therefore if we write $\left(d \alpha_{c} / d M\right)_{\text {equilibrium }}\left(=\mathrm{F}_{e}\right)=\mathrm{I} 20$ to 140 , it follows that $\delta M=\epsilon / \mathrm{F}_{\mathrm{e}}=0.0040$ to 0.0046 mass units per unit mass per minute, which is equivalent to a mass turnover rate of $24 \%$ to $28 \% / \mathrm{hr}$. This would be a minimum figure, since if we took a lower value for $F_{e}, \delta M$ would be correspondingly higher. What is more, only protein turnover is likely to lead to $\beta$-galactosidase synthesis, and since protein constitutes about $50 \%$ of the cell mass, a minimum protein turnover of about $50 \%$ to $60 \% / \mathrm{hr}$ would be required to account for all of the enzyme synthesis. This is out of the question, the results of Mandelstam (1957, 1960) and Willetts (1967) suggesting that protein turnover rates in non-growing organisms do not exceed $5 \% / \mathrm{hr}$. In addition the value taken as representing $\mathrm{F}_{e}$, I20 to I 40 , is much higher (by a factor of about $2 \cdot 8$ ) than the steady value in maltose adapted organisms, which itself requires explanation (it should be remembered that the m.g.t. of the organisms, during the first stages of growth in maltose, is about 2.5 times that of fully adapted organisms).

The above argument does not exclude the possibility of $\beta$-galactosidase synthesis via incorporation at a preferential (rather than a normal) rate, in contrast to de novo synthesis, but this would seem to be improbable on the following grounds. Protein turnover only occurs in the absence of growth (Mandelstam 1960). The hyperproduction of $\beta$-galactosidase in the present experiments continues into the period of active growth. There is no evidence of a change in mechanism at the onset of growth: $\alpha_{8}$ increases linearly from $t=0$ to $160 \mathrm{~min}$. This would seem to relegate synthesis via reincorporation to a secondary role, if it occurs at all.

\section{Organisms partially adapted to maltose}

Figure 4 gives results for organisms which had been transferred from glucose medium to maltose medium for two subcultures (ten generations) and then inoculated into $M+M T G$ medium. $\beta$-Galactosidase synthesis was followed for about six generations after the beginning of induction. The organisms were maintained in exponential growth by transfer to fresh medium after each assay. The values of $N, \Delta \alpha_{c}$ and $\Delta M$ given are calculated as the total values which would have been attained if the culture could have been kept in exponential growth without transfer to fresh medium (e.g. if $M$ in the original culture is 100 , and a twofold dilution is made whenever $M$ reaches $200 \Delta M$ at successive transfers will be $\left.100,300,700, \ldots\left(100 \times 2^{\mathrm{N}}-100\right)\right)$. Figure $4(b)$ 
shows that after growth for ten generations in maltose medium there is no measurable lag before logarithmic growth, although the growth rate (m.g.t. $=48 \mathrm{~min}$. at the beginning and $38 \mathrm{~min}$. at the end of the experiment) is not maximal. There is no appreciable preferential synthesis of $\beta$-galactosidase, on commencing induction, and the slope of the $\Delta \alpha_{c} v . \Delta M$ plot, 56 , is very little different from that eventually reached by fully adapted cells.

\section{Organisms stably adapted to lactose, gratuitously induced in maltose medium}

The adaptation of wild-type Aerobacter aerogenes to lactose medium can be stabilized by prolonged subculture in that medium. The details are given in Richards \& Hinshelwood (1962), but in brief such a stably adapted organism retains the ability to synthesise $\beta$-galactosidase during growth in maltose medium, and in the absence of

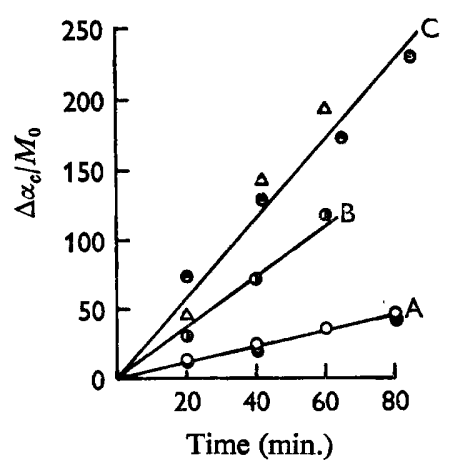

Fig. 3

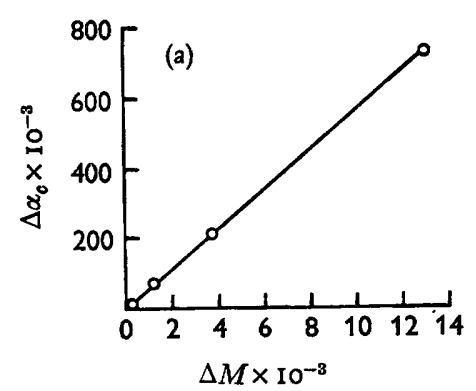

Fig. 4

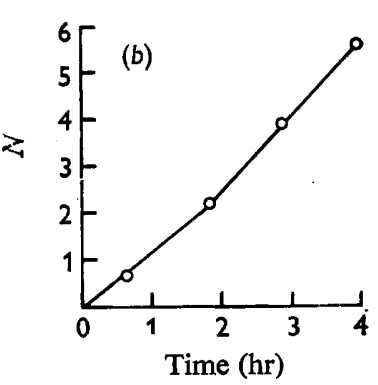

Time (hr)

Fig. 3. Amount of enzyme synthesized per unit cell mass, $\Delta \alpha_{c} / M_{0}$, during initial lag in maltose medium + MTG. A: Organisms unadapted to lactose medium. ( $O$ and $O$ represent different experiments) $\mathbf{B}$ and $\mathbf{C}$ : organisms stably adapted to lactose medium. B: washed organisms inoculated directly from lactose into maltose medium. C: organisms from lactose medium washed and incubated in medium without sugar $(\Theta)$ or without ammonium sulphate $(\Delta)$ for $150 \mathrm{~min}$. before inoculation into maltose medium.

Fig. 4. Gratuitous induction of a strain partially adapted to maltose. (a) Enzyme induction. There is an absence of preferential synthesis, and $d \alpha_{c} / d M(=56)$ is very little different from that characteristic of fully adapted organisms. $(b)$ Growth, expressed as number of generations $(N) \nu$. time, has not reached a rate characteristic of fully adapted organisms (m.g.t. at beginning of experiment $=48 \mathrm{~min}$., at end, $38 \mathrm{~min}$.),

inducer, to the extent of about IO-I $5 \%$ of the value in induced organisms. This compares with about $\frac{1}{2} \%$ in organisms not stably adapted, i.e. $\beta$-galactosidase synthesis is partially constitutive. In addition, stably adapted organisms grow immediately with maximal growth rate, on return to lactose medium after prolonged subculture in glucose or maltose medium, whereas unstably adapted strains develop lags and slow growth rates.

When such stably adapted organisms $\left(\mathrm{L}_{s}\right)$ are washed and transferred for the first time to maltose medium, growth in maltose follows the same pattern as in $G \rightarrow M$ transfers. $\alpha_{s}$ falls steadily to the value characteristic of the non-induced organisms (although enzyme synthesis continues, at a lower rate than in the induced organisms, throughout the period of dilution). 
If the transfer of $\mathrm{L}_{s}$ organisms is made to $\mathrm{M}+\mathrm{MTG}$ medium, there is a marked hyper-production of $\beta$-galactosidase, and $\alpha_{s}$ may rise as high as 700 (some five times as high as the normal value in $\mathrm{L}_{s}$ organisms growing exponentially in lactose, I20150). This is shown in Fig. 5 for: (a) organisms which were transferred direct from lactose to $\mathbf{M}+\mathbf{M T G}$, after a single wash in buffer, $(b)$ organisms which were taken from lactose, washed, and incubated at $40^{\circ}$ in medium without the nitrogen source, ammonium sulphate, for $150 \mathrm{~min}$., before inoculation into $\mathrm{M}+\mathrm{MTG},(\mathrm{c})$ organisms incubated as above, but in complete medium minus lactose.

These results would seem to exclude the possibility that any metabolic inter-
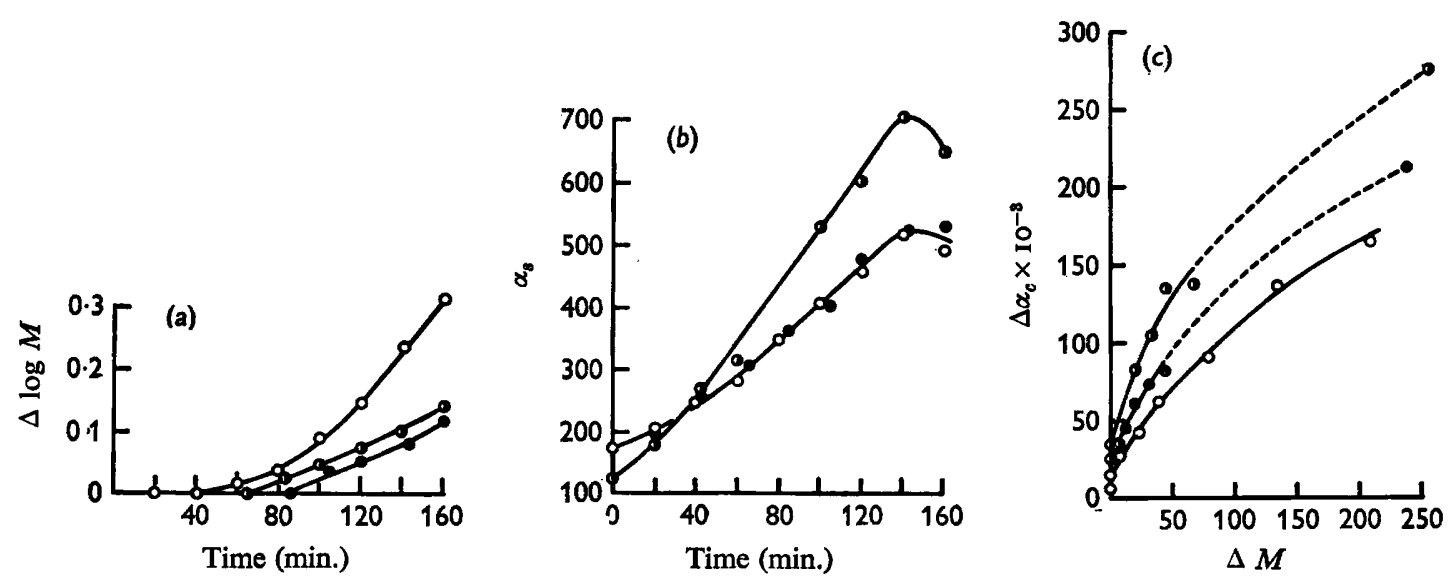

Fig. 5. A strain stably adapted to lactose $\left(\mathrm{L}_{\mathrm{g}}\right)$, washed and transferred to maltose $+\mathrm{MTG}$ medium for the first time. $O$, Direct transfer. O, Organisms incubated for $150 \mathrm{~min}$. in medium minus carbon source before inoculation into maltose medium. $\$$, Organisms incubated for $150 \mathrm{~min}$. in medium minus nitrogen source before inoculation into maltose medium. (a) Growth, in terms of $\Delta \log M$. (b) Specific enzyme activity. (c) New enzyme synthesis per unit volume of culture, against growth.

mediates carried over from the lactose medium might assist the preferential synthesis of $\beta$-galactosidase in M+MTG medium. In fact Fig. 3 (curves B and C) shows that enzyme synthesis during the earliest stages following transfer is most rapid (when measured in terms of enzyme units per unit mass per unit time) in the starved organisms. This suggests that, during starvation, ability to repress enzyme formation is lost, rather than ability to form enzyme on subsequent induction.

Arguments similar to those used in the previous section for $G \rightarrow M+M T G$ transfers can be applied here to show that there is a real preferential synthesis. It is harder to fix a maximum acceptable value for $F_{e}$. In none of the curves of Fig. 5 has a steady value of $d \alpha_{c} / d M$ been reached by the end of the experiment. $\alpha_{s}$ reaches a maximum value and begins to fall soon after the onset of exponential growth. It falls steadily during subculture in $M+M T G$ (see later). If we assume that the maximum value of $\alpha_{s}$ corresponds roughly to the maximum reasonable value of $F_{e}$, we obtain minimum values for mass turnover rate of $22 \%, 24 \%$ and $32 \% / \mathrm{hr}$ for unstarved organisms, organisms starved without nitrogen source, and without carbon source, respectively. These would represent an impossibly high protein turnover rate of around $50 \%$ per hour, if no preferential synthesis occurred. 


\section{Relation of hyper-production of $\beta$-galactosidase, in $L_{s}$ organisms, to state of adaptation to maltose}

The experiments described below, involving various strains of Aerobacter aerogenes, suggest that the preferential synthesis, and subsequent hyper-production, of $\beta$-galactosidase, following a medium change of the type $L \rightarrow M+M T G$, is a function of the degree of adaptation of the organisms to maltose, in much the same way as in $\mathbf{G} \rightarrow \mathbf{M}+\mathrm{MTG}$ transfers.

If $\mathrm{L}_{s}$ organisms, initially unadapted to maltose, are serially subcultured in $\mathrm{M}+\mathrm{MTG}$ medium, the value of $\alpha_{8}$ characteristic of mid-logarithmic phase bacteria falls steadily, as the organisms become adapted to maltose (Table I). In all serial subcultures, inoculum size $=\mathrm{I} / \mathrm{I} 00$ culture volume, so that on average each subculture is equivalent to $6 \cdot 7$ generations.

Table I. Values of specific activity of $\beta$-galactosidase $\left(\alpha_{8}\right)$ during the mid-logarithmic phase of organisms stably adapted to growth in lactose medium, being serially subcultured in maltose $+M T G$ medium

$\begin{array}{cc}\begin{array}{c}\text { No. of subculture } \\ \text { in M+MTG }\end{array} & \alpha_{8} \\ 1 & \approx 600^{*} \\ 2 & \approx 320 \\ 3 & \approx 290 \\ 8 & \approx 200 \\ 19 & 156 \\ 33 & 140 \\ 53 & 131 \\ 60 & 90 \\ 101 & 87\end{array}$

* Maximum value during exponential growth.

If the strain which is stably adapted to lactose is then subcultured in maltose until it is fully adapted (for 55 subcultures), before being grown in the presence of MTG, no preferential synthesis occurs (i.e. a plot of $\Delta \alpha_{c t} v . \Delta M$ gives a straight line through the origin), and the differential synthetic rate on gratuitous induction is approximately 150. This is much lower than the rate shortly after the change $\mathrm{L} \rightarrow \mathrm{M}+\mathrm{MTG}$ with organisms unadapted to maltose, and is in fact very close to the value reached in organisms grown for the same number of subcultures in M+MTG (see Table I).

When a strain fully adapted to maltose receives seven subcultures in lactose (conferring a partial, but not a stable, adaptation), before being washed and transferred to $\mathrm{M}+\mathrm{MTG}$ medium, the graph of $\Delta \alpha_{c} v . \Delta M$ is linear, passes through the origin, and has a slope of $50 \cdot 8$, characteristic of organisms adapted to maltose and growing in M+MTG with the equilibrium value of $\alpha_{s \text {. }}$

\section{DISCUSSION}

The results confirm the existence of a genuine 'preferential gratuitous synthesis' of $\beta$-galactosidase, in which enzyme is synthesized during the growth lag following transfer to maltose medium for the first time. The amounts of enzyme synthesized during the lag are far too large to be explained in terms of a non-preferential reincorporation 
of breakdown products into $\beta$-galactosidase molecules during protein turnover. This type of reincorporation was investigated by Mandelstam (1957), who suggested that it might operate in situations where other workers (e.g. Rickenberg \& Lester, 1955; Richards \& Hinshelwood, I962) have reported preferential synthesis.

The preferential synthesis obtained during the present work, in the lag period, is associated with hyper-production of the enzyme during the early stages of growth in maltose: i.e. the initial differential synthetic rate is maintained at an unusually high level for some time. In fact, there seems to be no reason to distinguish between enzyme synthesis during the lag and during early growth. Both are aspects of a general hyper-production of enzyme.

The hyper-production is, in a qualitative sense, not dependent on the previous induction history of the cells. It occurs both with organisms which have never been induced for $\beta$-galactosidase, previous to their transfer to $M+M T G$, and also with $\mathrm{L}_{\varepsilon}$ organisms transferred from lactose. It is, however, a function of the degree to which the organisms are adapted to maltose, being greatest with completely unadapted organisms, and diminishing progressively as adaptation proceeds.

An adequate and logical explanation of this behaviour seems to be possible in terms of the catabolite repression effect. The exposition of this effect given by Magasanik (I96I) requires that the effectiveness of catabolite repression should be a function of the speed with which the organisms can degrade the substrate, and hence the extent to which intermediates of the degradation will accumulate in the cellular environment. In general, the more quickly a particular carbon source can be degraded by the organisms, the more effective should repression be. Several examples of this have been discovered, in particular those discussed by Mandelstam (I962). The present results are an extension to a situation where the speed of degradation of a given carbon source changes with time-to an adaptative process in fact. The factor limiting the growth rate will be the rate of degradation of the new carbon source, maltose. The original hyper-production is a result of a breakdown in catabolite repression following transfer to a slowly degraded catabolite. As adaptation proceeds, and the carbon source becomes more rapidly metabolisable, the catabolite repression reasserts itself.

In the present experiments, $\beta$-galactosidase is gratuitous and plays no part in the adaptation. It is, however, tempting to think that the phenomenon of transient catabolite de-repression may be a general feature of processes involving adaptation to a new substrate. When the organism finds itself in a situation where rapid degradation of substrate molecules is impossible, there is an initial relaxation of the control of enzyme synthesis, owing to the depletion of a repressor molecule or molecules, rendering the organism particularly sensitive to the action of any inducer, which in turn might be used as a substrate. As the efficiency of the compound as substrate increases, so does its efficiency as repressor.

Part of this work was carried out during the tenure of a N.A.T.O. post-doctoral fellowship at the Physical Chemistry Laboratory, Oxford. The author wishes to thank the authorities of Norwich City College for a grant towards apparatus, and for some remission of teaching duties to allow him to pursue this research. He also wishes to thank colleagues at both institutions and at the University of East Anglia for helpful discussions. 


\section{REFERENCES}

Clark, D. J. \& MARR, A. G. (1964). Studies on the repression of $\beta$-galactosidase in Escherichia coli. Biochim. biophys. Acta $92,85$.

LoOMIS, W. F., JUN \& MAGASANIK, B. (I964). The relation of catabolite repression to the induction system for $\beta$-galactosidase in Escherichia coli. J. molec. Biol. 8, 417 .

LOOMIS, W. F., JUN \& MAGASANIK, B. (1967). The catabolite repression gene of the lac operon in Escherichia coli. J. molec. Biol. 23, 487.

Magasanix, B. (196I). Catabolite repression. Cold Spring Harb. Symp. quant. Biol. $26,249$.

MANDELSTAM, J. (1957). Turnover of protein in starved bacteria and its relationship to the induced synthesis of enzyme. Nature, Lond. 179, I 179.

MANDELSTAM, J. (1960). The intracellular turnover of protein and nucleic acids and its role in biochemical differentiation. Bact. Rev. 24, 289.

MANDELSTAM, J. (1962). The repression of constitutive $\beta$-galactosidase in Escherichia coli by glucose and other carbon sources. Biochem. J. 82, 489.

Moses, V. \& PREvost, C. (1966). Catabolite repression of $\beta$-galactosidase synthesis in Escherichia coli. Biochem. J. 100, 336.

NAKADA, D. \& MAGASANIK, B. (1964). The roles of inducer and catabolite repressor in the synthesis of $\beta$-galactosidase by Escherichia coli. J. molec. Biol. 8, 105.

NemHARDT, F. C. (1960). Mutant of Aerobacter aerogenes lacking glucose repression. J. Bact. 80, 536.

NemHARDT, F. C. \& MAGASANIK, B. (1956). Inhibitory effect of glucose on enzyme formation. Nature, Lond. 178,801 .

PALMER, J. \& MOSES, V. (1967). Involvement of the lac regulatory genes in catabolite repression in Escherichia coli. Biochem. J. 103, 358.

Prevost, C. \& Moses, V. (1967). Pool sizes of metabolic intermediates and their relation to glucose repression of $\beta$-galactosidase synthesis in Escherichia coli. Biochem. J. 103, 349.

RichaRds, N. \& HINSHELwoon, C. (I96I). Observations on the $\beta$-galactosidase activity of Bact. lactis aerogenes (Aerobacter aerogenes). Proc. $R$. Soc. B $\mathbf{5} 54,463$.

RichaRd, N. \& Hinshelwood, C. (1962). Gradual stabilization of adaptive $\beta$-galactosidase in Bact. lactis aerogenes. Proc. $R$. Soc. B I56, 20.

RICKENBERG, H. V. \& LESTER, G. (1955). The preferential synthesis of $\beta$-galactosidase in Escherichia coli. J. gen. Microbiol. 13, 279.

WILLETTS, N. S. (1967). Intracellular protein breakdown in non-growing cells of Escherichia coli. Biochem. J. 103, 453. 